She had been taking two-minim doses of tincture of digitalis in iced water with a few drops of brandy every half hour since the previous day. On the 22nd she had another bad night with decided increase of delirium and awful dyspnoea and some wandering during the day. 'The face, if possible, was more dusky. When seen at 9 P.M. she appeared to be moribund. The breathing consisted of a succession of short jerky sighs, the hands were icy cold, and the legs cold up to the knees. The radial pulse was fluttering and barely perceptible and there was whispering delirium. She was ordered one-tenth of a grain of morphine and stimulants with local application of warmth. She slept well after this and on the 23rd had rallied surprisingly in all directions. The improvement was maintained throughout the day, but towards erening the unfavourable symptoms returned. At midnight the patient awoke from a restless sleep and asked the nurse for some water, but before this could be given her she had fallen back dead.

It is matter for regret that an account of the post-mortem appearances is not available. The grouping of symptoms, however, and the character and development of the physical signs leave little doubt as to the nature of the case-blocking in the first instance of one of the branches of the pulmonary artery in the right lung with subsequent extension of the thrombus to other branches, pulmonary apoplexy, and death from cardiac failure. The chief interest of the case lies in the occurrence of this well-known series of events in a young girl who was apparently in perfect health. A diligent inquiry into the cause of the accident has been entirely negative in its results, and we have had to fall back on the hypothesis that the seeds of the mischief had been sown during the severe illness above referred to, possibly in the form of adherent thrombi in the right cavities of the heart, one of which became detached on June 16th.

Cliandos-street, $W$

\section{NOTE ON THE PICRIC ACID TEST FOR SUGAR.}

BY MARK MCDONALD, M.B. DUB.

WHILE carrying out some work on the urine some time since I came across certain reactions with the picric acid reduction test with which it is not generally credited. The importance which this test has acquired in the estimation, both qualitatively and quantitatively, of sugar in urine is sufficient excuse for bringing forward any points bearing on the subject.

When to a drachm of urine there are added a drachm of a saturated solution of picric acid, half a drachm of liquor potassæ, and one and a half drachms of water (as recommended by Sir George Johnson in the quantitative estimation of sugar), an orange-red colour is produced in the cold, due to the kreatinin present in the urine reducing the yellow potassium picrate to red picramate. On heating, this colour becomes more intense. Should the urine contain sugar in any quantity the effect of its presence will not be observed until the temperature reaches nearly $100^{\circ} \mathrm{C}$., at which point the colouration is evidently much intensified. If, however, before heating the mixture it be largely diluted-the addition of two ounces of water produces the effect most typically as a rule-the liquid is seen to gradually darken as it is heated, until it boils. Should the urine be normal it will be observed after a few seconds' boiling that the colouration has disappeared, and prolonged boiling does not bring it back; but if the liquid be cooled the colour gradually reappears, and this reaction may be repeated with the same solution any number of times. Should the urine contain sugar in any quantity the solution as before gradually darkens as heat is applied until it boils, when the colour (due to kreatinin) disappears, but if the boiling be prolonged in this case the solution again becomes coloured. This paradoxical reaction depends on the weakness of the solution in potassium picrate. Solutions of the latter when very weak are only with great difficulty partially reduced. The picramate produced by the action of kreatinin in the cold is on boiling reoxidised to picrate; sugar, on the other hand, reduces the weak picrate solutions only on prolonged boiling. A solntion of pure urinary kreatinin (prepared by Mr. S. Johnson's method) acts in a manner similar to a normal urine, more liquor potassæ being, of course, required, as Sir George Johnson pointed out. A dextrose solution on similar treatment caused reduction of the picrate only on very prolonged boiling.

It appears, then, that under certain conditions potassium picramate, when boiled in the presence of the substances by whose oxidation it has been produced from picrate, undergoes reoxidation at the expense of these oxidised bodies The conditions necessary are weakness of the picrate solution and capability of the ozidised bodies to easily undergo reduction. The latter condition is met with in the case of kreatinin. Other substances, as for example the alkaline sulphides, though they reduce in the cold, nevertheless do not reoxidise the picramate on boiling, as the products of their oxidation are very stable bodies. Dilution of the kreatinin solation also assists the reaction. Corresponding results are obtained if, instead of diluting the solutions before boiling, they are first boiled at the usual strength and then diluted with two ounces of water. In the case of normal urines and kreatinin solutions boiling removes the colour, while with sugar and alkaline sulphides no diminution takes place however prolonged the boiling may be. It is noteworthy in all these cases that when heat is applied after dilution the liquid assumes a distinctly darker colour before the boiling point is reached, showing that in the process of dilution and cooling some reoxidation of the picramate had taken place.

If the behaviour of normal urines and kreatinin solutions, on the one hand, and saccharine urines and dextrose solutions, on the other hand, be compared, their reducing power being equal, it is found that after prolonged boiling of the diluted solutions those which contain dextrose have a distinctly darker colour. The intensity of this final colour is proportional, too, to the quantity of dextrose present. It is possible by means of the different actions of kreatinin and sugar to determine whether the reducing effect of a urine is in part due to sugar. This can usually be effected by heating the diluted solution to nearly the boiling point in a long test-tube of small diameter, and then boiling the upper half of the liquid. The colour due to kreatinin disappears, and on boiling for a minute or two the upper part of the solution again darkens if sugar is present. It is important to remember that if the diluted liquid contains less than 1 part of dextrose in 10,000 no sugar reaction takes place, since, as Sir George Johnson noticed, its effect on alkaline picrate vanishes with higher degrees of dilution. This disturbance may be obviated by adding less water, or a dilute solution of potassium picrate may be prepared by mixing a drachm of saturated picric acid solution with half a drachm of liquor potassæ and adding eight and a half drachms of water. To one drachm of this solution one drachm of a twice or four times diluted and neutralised urine is added and the mixture treated as usual. In some cases it is necessary to compare the final colouration with that given by a kreatinin solution of equal reducing power.

Though I have occasionally during the last few months found this reaction of picric acid useful in deciding a diagnosis of glycosuria, I bring it forward less as a practical test than as a warning against a possible disturbance of the usual test for sugar.

Waterloo, Liverpool.

\section{THE DISCOVERY OF A BULLET LOST IN THE WRIST BY MEANS OF THE ROENTGEN RAYS.}

BY ROBERT JONES, F.R.C.S. EDIN.,

HONORAEY SURGEON TO THE ROYAI SOUTHERY HOSPITAL, LIVERPOOL; AND

OLIVER LODGE, F.R.S.,

PROFESSOR OF PHYSICS, UNIVERSITY COLLEGE, IIVERPOOL.

A BOY aged about twelve years was brought to me by Dr. Simpson of Waterloo, Liverpool, having shot himself in the left hand just above the deep palmar arch. The wound was enlarged, but the bullet could not be found, and it was thought injudicious to prolong the search in vien of the important structures in the vicinity unless one possessed a clue to its position. Professor Lodge kindly consented to take a photograph and the position of the bullet was rery clearly outlined. It lies against the base of the third meta. carpal bone over its articulation with the os magnum. This 
is, I think, the first photograph taken of a bullet embedded in a wrist-in this case considerably thickened as a result of inflammation. It will now be quite easy to extract it but it is thought better to await subsidence of inflammatory action. The photograph was taken in the presence of Mr. Houlgrave, who succeeded Dr. Simpson in the treatment of the case. Arrangements are being competed for fitting up a department for the employment of Roentgen's rays at the Royal Southern Hospital.

Note by Professor OLIVER LongE.-The patient was brought to my laboratory by Mr. Robert Jones, with a pellet of lead lost in his left hand or forearm. Two

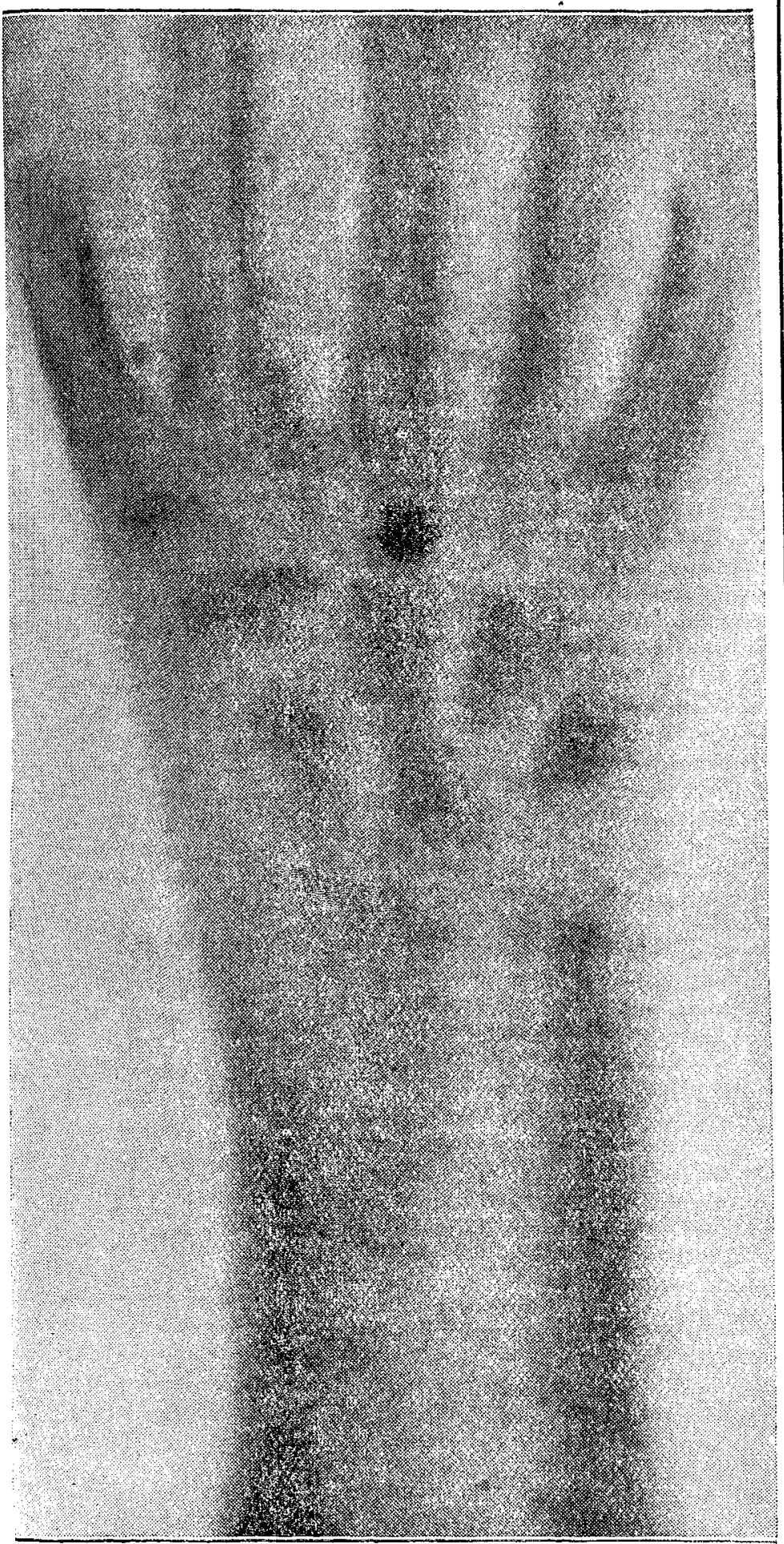

Roentgen radiograph of the left wrist of a lad aged twelve years, showing a small bullet which had been lost in it. The bullet is located between the base of the middle metacarpal bone and the os magnum. Taken by Professor Oliver Lodge, F.R.S., after two hours' exposure to a well exhausted home-made vacuum tube excited by a small ordinary coil. The sensitive plate was an Ldwards's isoscreened from light by sheet aluminium.

preliminary short exposures to Roentgen rays indicated that the metal was not in a fleshy part readily penetrable by those rays, but was probably embedded among the bones of the wrist. The difficulty consisted in the opacity of those bones. I therefore took a tube with large electrodes made and exhausted by my assistant, Mr. E. E. Robinson, containing potash, so that its vacuum could be adjusted to the best value, and arranged a magnet to concentrate the inside cathode-rays on a definite part of the glass, which phosphoresces and acts as the source of the rays. the rays spreading from each point of the glass in all directions. The boy was comfortably seated at a table with his palm down on an aluminium-protected Edwards' isochromatic half-plate, nine inches vertically below the vacuum tube, and rather more than two hours' exposure was given. The coil used was an ordinary Ruhmkorff, giving about two inches spark at most, and it was excited by five storage cells. The vacuum was such that a one-inch spark in air was usually preferred to the tube. The result was to bring out the wrist-bones clearly and to show the position of the pellet.

Liverpool.

\section{LATENCY IN SYPHILIS.}

By ARTHUR H. WARD, F.R.C.S. ENG.,

GURGEON TO OUT-PATIENTS, LONDON LOCK HOSPITAL, AND SURGICAT REGISTRAR TO ST. GEORGE'S HOSPITAL.

In the admirable paper read by Mr. Ernest Lane before the Royal Medical and Chirurgical Society on Dec. 10th, 1895, many cases were brought forward in proof of the fact of latency in syphilis; but neither in the paper nor in the discussion which followed was much said as to the causation of that latency which all admitted. I believe that some reasons may be brought forward to account for both the apparent and real latency of syphilis. By apparent latency I mean a period during which the re are no visible lesions although the microbes are "doing their work," as Mr. Hutchinson put it, more or less actively. Real latency, however, I take to mean a period during which the microbes are absolutely inactive. In a former paper ${ }^{1}$ I ventured to state a working hypothesis to account for the causation of syphilis and now state it thus: (1) Syphilis is associated with the growth of a microbe in the organism; (2) this microbe produces a toxine, which is the active cause of the phenomena of syphilis ; and (3) this toxine when entering the human body in small and slowly increasing quantities establishes immurity. It would seem to be hardly necessary at the present time to adduce arguments in favour of the existence of such a microbe and consequent active toxine, since the microbe is generally admitted, while the activity of so many other microbes has been shown to be due to the formation of toxines. However, a somewhat striking evidence of its presence is furnished by the experiments of Straus and Tessier, who found that strong reactions occurred in cases of secondary syphilis when tuberculin injections were made; and that in a case of rupia an inflammatory areola formed round the lesions "identical with that occurring in cases of lupus" when injected. ${ }^{2}$ "It will be admitted that reaction following tuberculin injection certainly indicates the presence of the tubercle bacillus and its toxine, consequently a similar reaction in a case of syphilis. indicates the presence of an analogous microbe and toxine in that disease.

The incubation period of syphilis is one of apparent latency. I think it is due to the circumstance that the microbe is a slow-growing one, as the course of the disease indicates, and also to the fact that only a few microbes can possibly be implanted at the moment of infection. Consequently these take time to grow into a colony large enough to produce an appreciable amount of toxine. Induration, the result of the chemiotaxic action of the toxine, can, therefore, not occur at once; but when a growth of three weeks or so has taken place toxine enough to attract round cells into the colony can well be formed. These cells when they have accumulated sufficiently distend the inter-cellular spaces and cause the indurated papule, which grows steadily with the peripheral growth of the colony and generally breaks down in the centre forming the indurated sore. It is to be noted that primary tuberculous inoculations also produce indurated lesions. The next period of apparent latency in the course of syphilis is the so-called second incubation period between the formation of the primary sore and the outbreak of secondaries. This period, I think, must have been in Mr. Hutchinson's mind when he spoke of microbic activity being

1. Thr Laxcer, Sept. 1Cth, $1892 . \quad 2$ Medical Week, 1893, p; 399. 